Kompass

Pneumologie

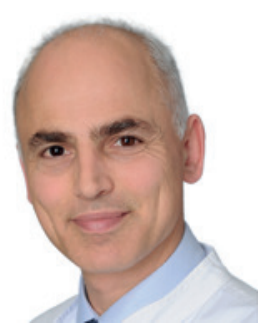

\section{Khosro Hekmat}

Klinik und Poliklinik für Herz- und

Thoraxchirurgie, Universität zu Köln, Köln, Deutschland

\title{
Chancen und Risiken der Thoraxchirurgie in einem multimodalen Therapieansatz beim fortgeschrittenen nicht kleinzelligen Lungenkarzinom
}

Lungenkrebs ist weltweit die häufigste Ursache für krebsbedingte Todesfälle bei beiden Geschlechtern. Nicht kleinzelliger Lungenkrebs (non-small cell lung cancer, NSCLC) macht etwa 85\% aller Lungenkrebsdiagnosen aus. Im Bereich der Thoraxonkologie haben die jüngsten klinischen Studien zur multimodalen Therapie in Kombination mit neu entwickelten Wirkstoffen, einschließlich der molekularen zielgerichteten Therapie und der Immuntherapie, eine hohe pathologische Ansprechrate gezeigt, was für einige Patienten mit fortgeschrittenem NSCLC bessere Heilungschancen bieten könnte.

Die Präzisionsmedizin in Form einer optimalen multimodalen Therapie, die Bestrahlung und systemische medikamentöse Therapie mit einem optimalen Zeitpunkt für die chirurgische Resektion kombiniert, könnte bei Patienten mit fortgeschrittenem NSCLC sogar zu einer vollständigen Heilung beitragen. Lange beschränkte sich die chirurgische Therapie des NSCLC auf die Stadien I-III. Oligometastasierte NSCLC-Patienten sind jedoch ganz aktuell in die S3-Leitlinie des Lungenkarzinoms aufgenommen worden. In den jüngsten Studien für NSCLC werden multimodale Therapieansätze für lokal fortgeschrittene, resektable Tumoren empfohlen. Die neo- adjuvante Therapie wurde der chirurgischen Resektion beim fortgeschrittenem NSCLC hinzugefügt, um möglichst auch hier eine vollständige Heilung zu erreichen. In den letzten Jahrzehnten haben neu entwickelte Medikamente für NSCLC mit Treiber-Onkogenen wie EGFR (epidermal growth factor receptor), ALK (anaplastic lymphoma kinase), ROS-1 (reactive oxygen species-1), BRAF ( $v$-raf murine sarcoma viral oncogene homolog B1) und die Tumorzellexpression von PD-L1 (programmed death ligand 1) zusätzliche therapeutische Optionen geschaffen, die in klinischen Studien nachgewiesen wurden. Mit dem Aufkommen der Immuncheckpoint-Inhibitoren ist die Rolle der Thoraxchirurgen bei der Entscheidung über die geeignete Strategie zur vollständigen Heilung von Patienten mit fortgeschrittenem Lungenkrebs noch wichtiger geworden. Folglich werden wir neue Erkenntnisse über geeignete neoadjuvante Behandlungsansätze haben. Darüber hinaus werden Tyrosinkinase-Inhibitoren je nach Vorhandensein von Onkogen-Treibern eine wichtige Rolle in der Präzisionsmedizin spielen.

Das Standardmedikamentenregime mit einer Platindoublette bleibt vorerst weiterhin bestehen. Nachdem die Tumorzellen durch eine multimodale Therapie, einschließlich neu entwickelter Induktionstherapieformen und einer vollständigen chirurgischen Ro-Resektion, die manchmal sogar komplexe Manschettenresektionen erfordert, so weit wie möglich eliminiert wurden, könnte fortgeschrittener Lungenkrebs vollständig geheilt werden.

Umgekehrt wurden auch negative Ergebnisse aus neoadjuvanten Immuntherapiestudien berichtet, und Thoraxchirurgen sollten diese Studiendesigns sorgfältig kennen und analysieren. Multimodale Therapien, einschließlich ausgedehnter Operationen oder minimalinvasiver Behandlungen, müssen so geplant werden, dass ein Gleichgewicht zwischen den Risiken und Vorteilen der personalisierten Therapie und der molekularen biomarkerbasierten präzisen Medizin besteht. Thoraxchirurgen müssen die Operabilität eines Tumors auch nach neuen komplexen neoadjuvanten Therapieformen beurteilen können, da sich die systemischen Krebstherapien extrem rasch entwickeln. Die Zukunft für neue Therapieansätze beim NSCLC bleibt spannend.

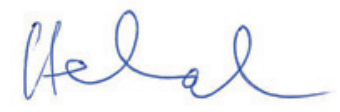

Prof. Dr. Khosro Hekmat 